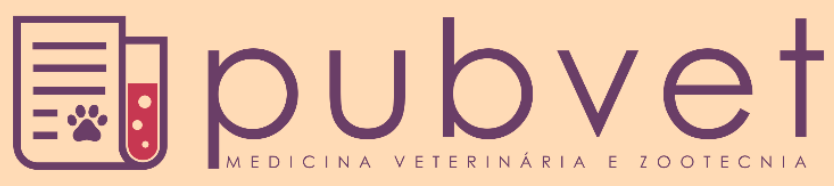

https://doi.org/10.31533/pubvet.v15n05a813.1-9

\title{
Enriquecimento ambiental em zoológicos
}

\author{
Beatriz Bezerra Morezzi ${ }^{1 \oplus}$, Izabella Sponchiado Alves $^{1 \oplus}$, Larissa Ayashi Kawanichi $^{1 \oplus}$, Maria \\ Clara Silva Bergamo ${ }^{1}{ }^{\ominus}$, Martina Galeriani Pirasol ${ }^{1} \bullet$, Milena Ingles dos Santos $^{1}{ }^{\circ}$, Fernanda \\ de Paula Roldi Vieira ${ }^{2}$, Mauro Henrique Bueno de Camargo ${ }^{3 *}$
}

${ }^{I}$ Graduanda em Medicina Veterinária na Universidade Estadual de Maringá, Departamento de Medicina Veterinária. Umuarama-PR. ${ }^{2}$ Mestre em Medicina Veterinária

${ }^{3}$ Docente em Medicina Veterinária na Universidade Estadual de Maringá, Departamento de Medicina Veterinária, Umuarama-PR.

*Autor para correspondência, E-mail: maurohbc@gmail.com

Resumo. Os zoológicos foram criados com objetivos que divergem da realidade presente, voltados atualmente para a conservação de espécies, educação ambiental e pesquisas científicas, não unicamente para entretenimento do homem. Visando melhorar a vida dos animais mantidos em cativeiro, o enriquecimento ambiental promove estímulos que proporcionam a interação animal com o meio e com outros seres, possibilitando expressar comportamentos comuns à sua espécie, aumentando, deste modo, o grau de bem-estar. Pode ser aplicado em cinco diferentes métodos: social, físico, sensorial, cognitivo e alimentar, tendo em vista o objetivo a ser aplicado, comportamentos e hábitos de cada espécie. O recinto deve apresentar as necessidades que o cativo exige, assemelhando-se ao máximo com o habitat natural e a realidade em vida livre. A falta ou aplicação ineficiente do enriquecimento pode acarretar inúmeros problemas, como aumentar índices de depressão, ansiedade, estresse, reduzir taxas reprodutivas e entre outros que serão apresentados de forma mais aprofundada neste trabalho. $\mathrm{O}$ presente estudo abordou um compilado de bibliografias que realçam o papel dos zoológicos com o decorrer dos anos e a relevância do enriquecimento ambiental nos recintos agregado ao bem-estar animal e melhor qualidade de vida.

Palavras-chave: Bem-estar, cativeiro, depressão, estresse, estímulos, recinto

\section{Environmental enrichment in zoos}

Abstract. Zoos were created with objectives that diverge from the present reality, currently focused on the conservation of species, environmental education and scientific research, not only for the entertainment of man. In order to improve the lives of animals kept in captivity, environmental enrichment promotes stimuli that provide animal interaction with the environment and with other beings, making it possible to express behaviors common to their species, thus increasing the degree of well-being. It can be applied in five different methods: social, physical, sensory, cognitive and food, in view of the objective to be applied, behaviors and habits of each species. The enclosure must present the needs that the captive requires, resembling to the maximum with the natural habitat and the reality in free life. The lack or inefficient application of enrichment can lead to numerous problems, such as increasing rates of depression, anxiety, stress, reducing reproductive rates and among others that will be presented in more depth in this work. The present study covered a collection of bibliographies that highlight the role of zoos over the years and the relevance of environmental enrichment in the enclosures added to animal welfare and better quality of life.

Keyword: Well-being, captivity, depression, stress, stimulus, enclosure 


\section{Introdução}

Foi pela civilização egípcia que o costume de expor animais deu início, passando assim, pelas gerações, até chegar ao que se conhece hoje, como zoológico. Os primeiros zoológicos foram criados com animais capturados do meio livre e com recintos precários (Sanders \& Feijó, 2007). Atualmente, a maioria dos zoológicos abrigam animais resgatados de maus tratos, além de animais ameaçados de extinção, com o objetivo de conservar as espécies (Damasceno, 2018; Silva et al., 2015). No entanto, os recintos onde são mantidos os animais muitas vezes são inadequados, o que proporciona comportamentos incomuns à espécie, como agressividade excessiva, estresse, estereotipias e, também, inatividade, condições que não seriam encontradas em habitat natural (Oliveira \& Carpi, 2016).

Para amenizar essa problemática que acompanha os zoológicos, é utilizado o enriquecimento ambiental, o qual tem a finalidade de melhorar a qualidade de vida dos animais de cativeiro, usando estímulos ambientais corretos, que simulam acontecimentos reais, criando um ambiente com maior complexidade e interatividade (Oliveira \& Carpi, 2016). Os métodos de enriquecimento são usados de acordo com características específicas de cada animal, tendo como meta assemelhar o recinto, cada vez mais, com o ambiente natural da espécie (Bosso, 2011). Desse modo, gerando um bem-estar físico e psicológico ao animal (Oliveira \& Carpi, 2016).

Por fim, esse trabalho tem como objetivo mostrar a história da fundação dos zoológicos e, principalmente, demonstrar a importância do enriquecimento ambiental nos recintos, para que assim, os animais possuam uma melhor qualidade de vida, sem passar por alterações físicas e comportamentais.

\section{Zoológicos}

Possuir grandes coleções de animais, na antiguidade, era sinal de força e poder (Silva, 2019; Singer, 2013). Esse costume foi passando por gerações, até que os primeiros zoológicos fundados capturaram seus animais oriundos de circos, outros eram compostos por animais para estudo. Como precisavam manter esses abrigos, foram abertos ao público para obter alguma renda. A necessidade de procurar por animais novos para o entretenimento fez com que muitos deles fossem capturados sem nenhum cuidado ou controle, levando muitos à extinção (Sanders \& Feijó, 2007).

Os primeiros jardins zoológicos modernos surgiram no século XIX. Caracteriza-se como jardim zoológico, um espaço com coleções de animais silvestres em cativeiro, ou semiliberdade, sendo expostos. De acordo com a Lei Federal 7.173/83, todos os estabelecimentos devem atender as condições mínimas de habitabilidade, sanidade, segurança, necessidades ecológicas e manejo, bem como tratamentos indispensáveis e proteção do público (Silva, 2019).

A partir da década de 90, esses jardins foram e estão sendo diretamente relacionados com a conservação animal, educação ambiental e pesquisa científica e não somente ao entretenimento humano (Dias, 2003; Sanders \& Feijó, 2007). Um dos principais objetivos é proporcionar aos animais, um

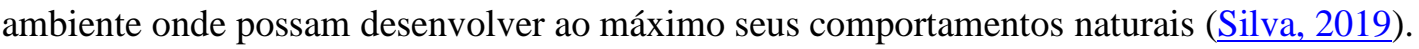

No século XX, as discussões sobre o bem-estar animal foram iniciadas (Ferreira et al., 2020), visando garantir condições satisfatórias às necessidades físicas e mentais dos animais (Chiquitelli Neto et al., 2011). A OIE (Organização Mundial da Saúde Animal) (2019), enquadra o bem-estar animal (BEA) àquele que possui acesso às cinco liberdades, sendo elas: liberdade da fome, sede e desnutrição; do medo e angústia; do desconforto físico e térmico; da dor, lesões e doenças; e ter liberdade para expressar seus padrões normais de comportamento.

\section{Enriquecimento ambiental (EA)}

Enriquecimento ambiental (EA) é uma forma de buscar a melhor qualidade de vida para um animal mantido em cativeiro, mantendo-o mais ativo e diminuindo suas estereotipias, ou seja, algum comportamento que ele não teria se estivesse em seu habitat natural (Damasceno, 2018; Massari et al., 2018; Silva et al., 2015). Podendo ter configurações sociais ou físicas, o EA não consiste em apenas colocar objetos no recinto, compreende também o tamanho do mesmo, o tipo de forragem (revestimento do local onde o animal dorme) e contato social com outros animais (Massari et al., 2018). 
Em cativeiro, esses animais precisam se exercitar com estímulos semelhantes aos encontrados lá fora, pois, uma vez que foram retirados de seu ambiente, foi retirada também a necessidade de lutar por sobrevivência e, portanto, ficam inativos, perdendo grande parte de seus comportamentos instintivos devido à falta de complexidade do recinto do zoológico (Damasceno, 2018; Silva et al., 2015). A existência de estereotipias está diretamente relacionada ao baixo grau de bem-estar, desencadeadas pelo estresse da privação de liberdade, comprometendo o crescimento, a capacidade imunológica e reprodutiva, facilitando enfermidades e aumentando a mortalidade (Artigas \& Fischer, 2019).

Ambientes enriquecidos contribuem para o BEA, melhora a saúde psicológica do animal, reduzindo o estresse e prevenindo comportamentos anormais. Também facilita o manejo e promove educação ambiental aos visitantes do zoológico. Os animais que são criados nestes ambientes raramente apresentam distúrbios comportamentais; pelo contrário, agem de modo semelhante à sua natureza, aumentando a taxa reprodutiva e são mais suscetíveis às adaptações, contribuindo diretamente à reintrodução de alguns desses animais ao habitat natural (Garcia \& Bernal, 2015; Leira et al., 2017).

É comprovado que o EA tem efeitos significativos se tratando de emoção, cognição, comportamento, fisiologia, peso corporal, reprodução, secreção hormonal, desenvolvimento da prole e ainda controle do estresse por estar preso no mesmo local por muito tempo (Massari et al., 2018). Tem sido cada vez mais reconhecido como uma maneira de melhorar o bem-estar desses animais, providenciando estímulos que satisfazem as necessidades espécie-específica de cada um (Damy et al., 2010).

\section{Métodos de enriquecimento ambiental}

É possível definir o comportamento típico de uma espécie por meio de características como a busca por alimento, acasalamento, fuga e refúgio. Sendo assim, as expressões individuais são fundamentais para evitar o tédio e estresse, além de contribuir para o aumento das chances de reinserção do animal na natureza (Silva \& Macêdo, 2014).

A escolha de atividades a serem inseridas no recinto está intimamente relacionada ao conhecimento comportamental da espécie e aos diferentes hábitos dos indivíduos (Costa, 2015) É imprescindível que o planejamento do EA seja focado em um objetivo, e somado ao devido acompanhamento dos resultados à longo prazo, para assim observar respostas benéficas ou não frente as mudanças estabelecidas (The Shape of Enrichment, 2020). Antes de definir o EA a ser incluído, é preciso atenção em aspectos como o tipo de alimentação, a segurança dos objetos oferecidos (de modo que não causem danos ao público e ao próprio animal), a quantidade ofertada suficientemente para todos os indivíduos do ambiente, o tempo de permanência da atividade interativa, e até as limitações e restrições do animal (Almeida et al., 2008).

\section{Enriquecimento físico}

Está associado às características estruturais do recinto e a presença de elementos que se assemelhem ao habitat natural, como a climatização, presença de ponto de fuga e a inserção de objetos para interação (troncos, pedras, água e folhagens), como retratado na figura 1 (Bosso, 2011).

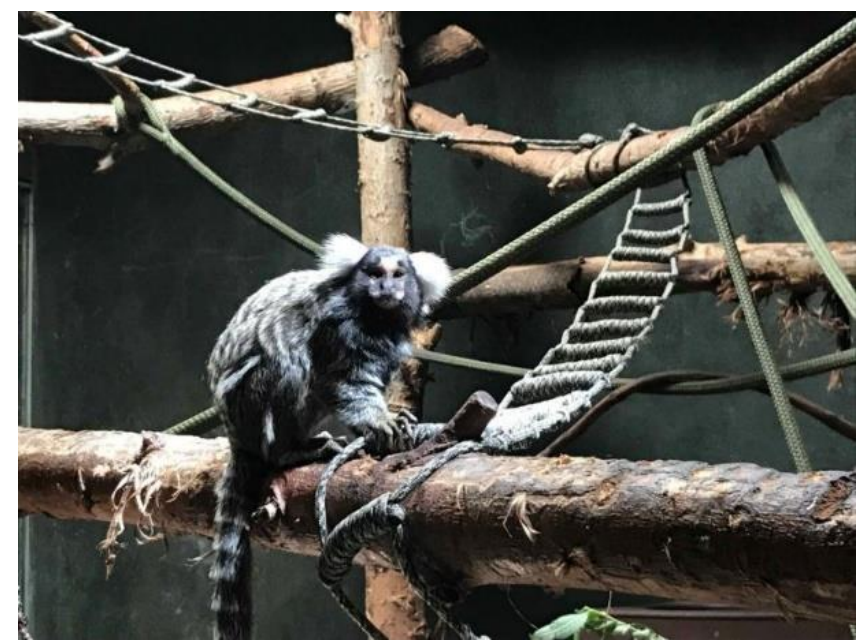

Figura 1. Exemplo de enriquecimento físico com adição de troncos e cordas no recinto. Fonte: Jones (2019). 


\section{Enriquecimento sensorial}

É definido como a introdução de atividades que explorem os sentidos do animal, seja de modo auditivo, com a adição de sons da natureza; gustativo, diversificando a variedade dos alimentos oferecidos; ou até mesmo olfativo, com a presença de excremento de outros animais e essências com cheiros específicos. Já os sentidos de visão e tato podem ser desenvolvidos com a inclusão de diferentes substratos e objetos de interação, como visto na figura 2 (Almeida et al., 2008).

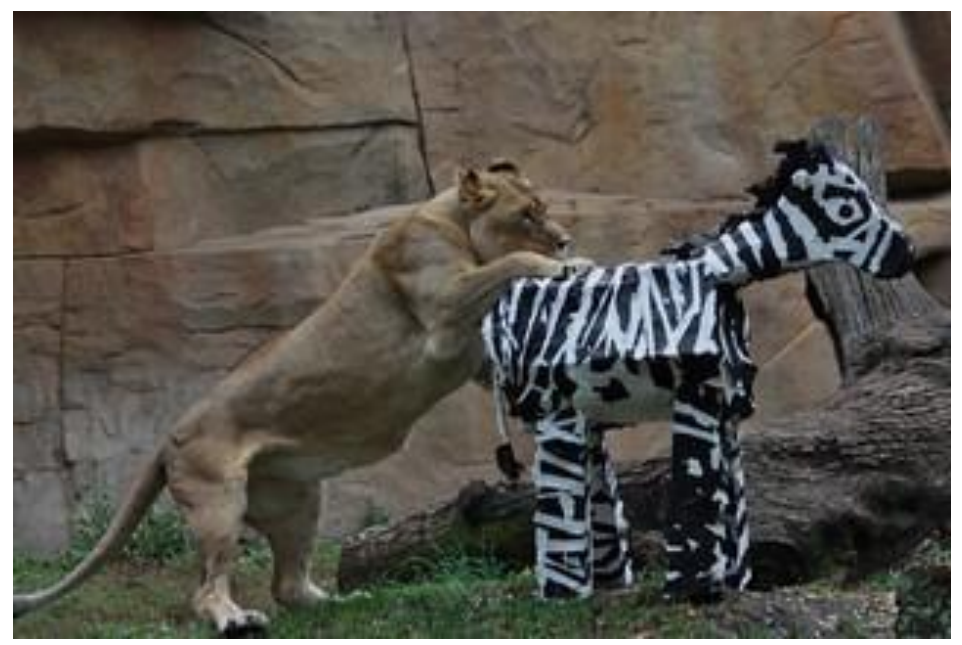

Figura 2. Exemplo de enriquecimento sensorial tátil e visual, leoa interagindo com objeto que simula presa. Fonte: Flickr (2014).

\section{Enriquecimento cognitivo}

Elementos que estimulam a capacidade intelectual e desenvolvem a característica de exploração, utilizando equipamentos mecânicos, atividades físicas e peças de encaixe; bem exemplificado pela figura 3 , como uma forma de jogo e recompensa para o animal (Almeida et al., 2008).

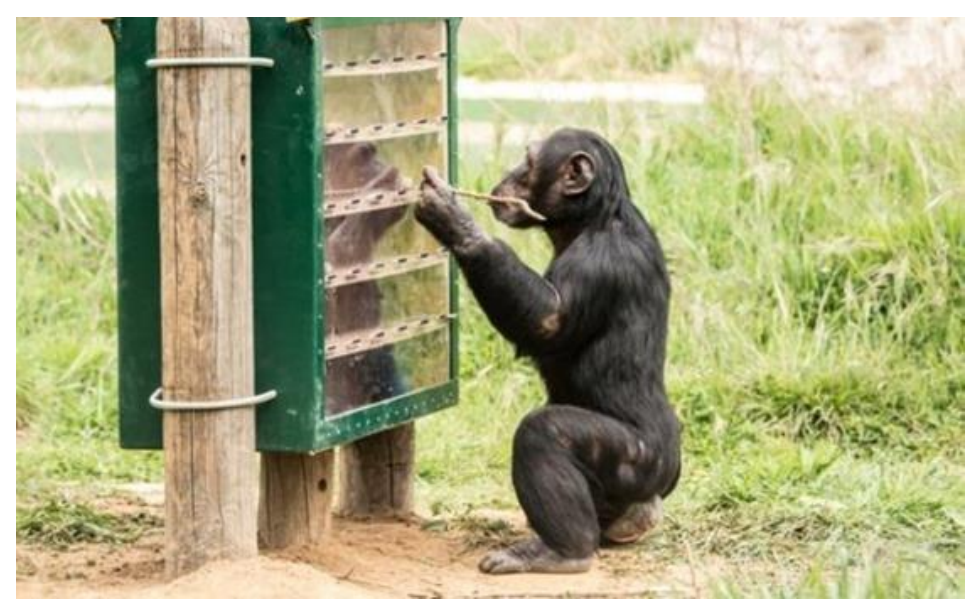

Figura 3. Exemplo de enriquecimento cognitivo, jogo para chimpanzés. Fonte: Fundación Mona.

\section{Enriquecimento social}

Fornecimento de uma inter-relação entre espécies iguais ou diferentes (visível na figura 4), para promover a convivência que o animal teria em condições naturais (Bosso, 2011). Em locais que o convívio de animais é inviável, pode-se utilizar de meios que simulem esta realidade, representada pela figura 5 por meio de espelhos no recinto de uma espécie que naturalmente vive em grupos maiores (Bosso, 2011). 


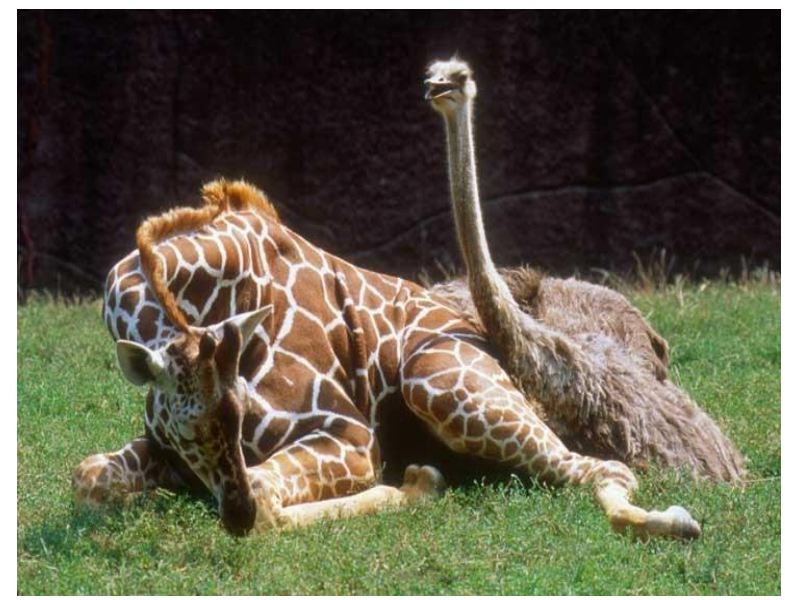

Figura 4. Exemplo de enriquecimento social, girafa e ema no mesmo recinto. Fonte: Saint Louis Zoo.

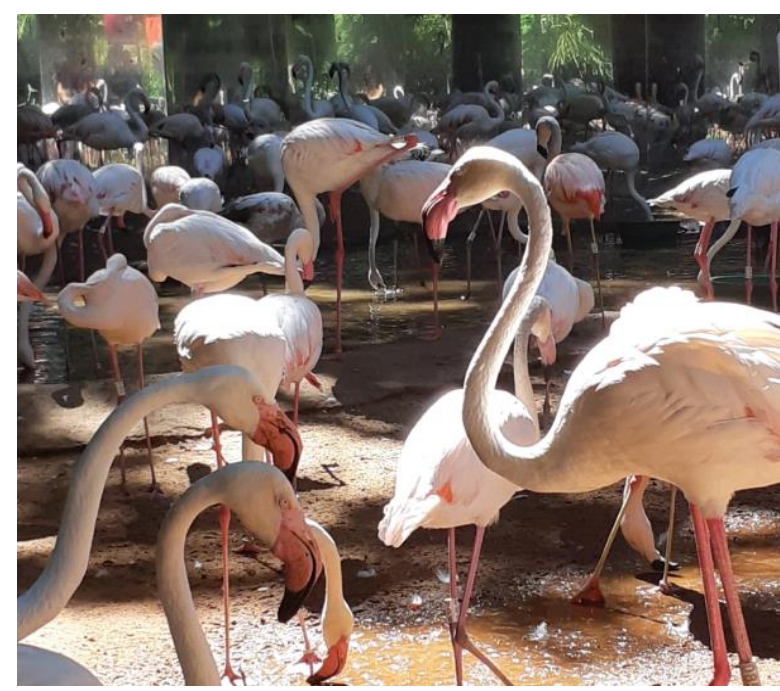

Figura 5. Exemplo de enriquecimento social artificial, utilização de espelhos em recinto de flamingos.

\section{Enriquecimento alimentar}

É a modificação nos meios de oferecimento da dieta, que pode ser disponibilizada em diferentes períodos do dia, alternando o tipo de alimento, frequência, local, ou como visto na figura 6 , em que a forma de apresentação, e a dificuldade no grau de obtenção, são proporcionados em conjunto (Almeida et al., 2008).

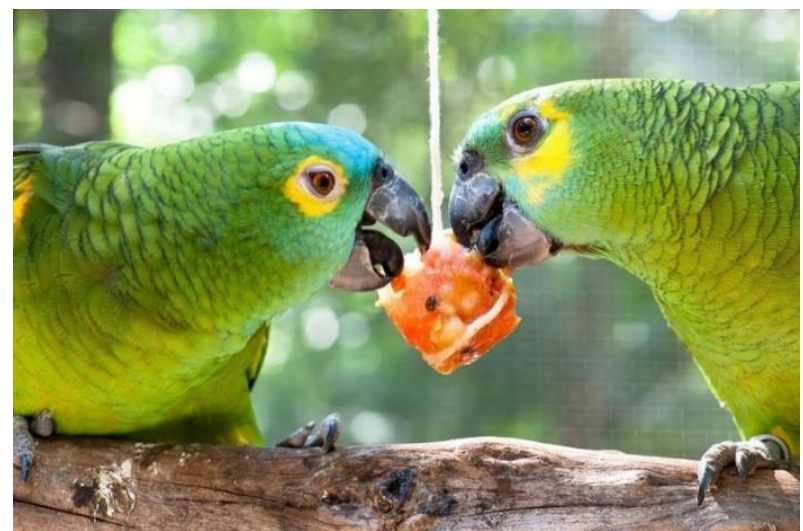

Figura 6. Exemplo de enriquecimento alimentar, disponibilização de frutas congeladas para aves. Fonte: Foz Portal da Cidade (2019). 


\section{Manejo irregular}

O enriquecimento ambiental feito de maneira errônea faz com que os animais não exerçam seus comportamentos habituais. Na natureza, possuíam estímulos diários como se esconder, caçar, buscar abrigo, proteger-se e se reproduzir. Já em cativeiro, retém-se em um espaço diminuto em comparação ao habitat natural, com falta de incentivos em busca do próprio alimento ou em esconder-se de predadores (Leira et al., 2017). Além de que, os que estão cativos em zoológicos, podem sofrer com a habitual visitação de público, com o sentimento de solidão, sons altos, luz e cheiros não apropriados (Azevedo \& Barçante, 2018).

Os hábitos incomuns decorrentes são distinguidos em quantitativo e qualitativos, sendo o primeiro uma tendência de repetir o comportamento inúmeras vezes, como lambidas, podendo ocasionar em dermatite. $\mathrm{E}$ os qualitativos são gestos que não são vistos em habitat natural, chamados de comportamentos estereotipados, são aqueles sem propósito e realizados com frequência (Leira et al., 2017). Em suma, o estresse pode ser classificado em agudo ou crônico. O primeiro sendo benéfico, por aumentar a excitabilidade resultando em atividades fisiológicas e psicológicas benevolentes, ajudando na liberação de norepinefrina e epinefrina, hormônios contribuintes na reprodução animal. Entretanto, o estresse crônico atua reduzindo a libido, podendo impedir o acasalamento e consequentemente a reprodução, levando ainda a altas doses de cortisol no organismo, resultando em atrofia tecidual e comportamentos anormais (Silva, 2019).

Além disso, manejar o recinto de maneira inapropriada, propicia agentes estressores, sendo eles os somáticos, relacionados a ruídos, cheiros, ambiente estranhos, temperatura muito elevada ou baixa; psicológicos, devido ao sentimento de ansiedade e apreensão; e também aos comportamentais, resultantes de brigas por dominação territorial, aglomeração de animais cativos juntos no mesmo recinto e ausência de privacidade (Leira et al., 2017). É possível ver comportamentos agressivos marcados com automutilação, perda de penas e pelos, hábitos contínuos de morder ou lamber, ingestão de excrementos ou terra. Sendo possível que haja a hiperagressividade, canibalismo, matança de recém-nascidos, ou a ocorrência de aborto em casos de estresse elevado (Leira et al., 2017). O organismo é capaz de suportar fatores estressantes até determinado limite, sem haver nenhum dano, porém quando ultrapassa essa capacidade, o animal passa a ter perda de homeostase, baixas condições de bem-estar e acarreta distúrbios comportamentais (Telles et al., 2015), como o pacing (andar de um lado para o outro repetidamente, é considerado um comportamento estereotipado). Essa problemática, ocorre devido a previsibilidade e falta de complexidade do ambiente, assim perdendo seus instintos e comportamentos naturais (Damasceno, 2018; Silva et al., 2015).

Os animais vertebrados ao se integrarem em ocorrências que não podem ser previstas, sofrem de mudanças fisiológicas e em sua conduta. Assim, acontecimentos imediatos resultam no "estágio de emergência" ocasionando modificações endócrinas e metabólicas, dispondo de frequência cardíaca e respiratória elevada, com liberação de glicose em função de haver energia disponibilizada pelo fígado, tornando o animal a entrar em homeostase posteriormente (Silva, 2019).

\section{Abordagem}

Como forma de solucionar ou pelo menos conter essa situação, o EA conta com diversas atividades e modificações no ambiente, com o intuito de proporcionar o bem-estar do animal. Além de poder ser aplicado em qualquer tipo de espaço, mesmo aqueles pequenos e pobres de estímulos, a fim de diminuir o tempo de inatividade. Animais criados em recintos enriquecidos, têm maior desenvolvimento, capacidade de aprendizagem e menos ansiedade (Damasceno, 2018; Silva et al., 2015). É uma abordagem de sucesso, justamente pelos animais diminuírem a frequência de comportamento de apatia, passando mais tempo interagindo com o enriquecimento. Além de ter várias maneiras de implementálo, havendo métodos simples e acessíveis, podendo ser introduzidas em qualquer recinto (Damasceno, 2018; Silva et al., 2015).

Alguns pontos devem ser levados em consideração ao introduzir o EA: deve-se usar corretamente os estímulos ambientais, observar os comportamentos e parâmetros fisiológicos do animal, além de usar todas as informações que possui sobre a biologia da espécie, deste modo a obtenção de efeitos positivos, como o bem-estar psicológico e físico do animal será alcançada (Oliveira \& Carpi, 2016). 


\section{Observações e resultados de técnicas de experimentos}

Visto as necessidades, os empregos das técnicas de EA são acessíveis à maioria dos zoológicos, devido sua simplicidade, seu baixo custo e não requerer treinamento específico para sua aplicação (Manfrim et al., 2017). Os resultados de experimentos de enriquecimento ambiental realizados em zoológicos tendem a ser sempre muito positivos, comoé mostrado por Ricci et al. (2018), onde é relatado que em cativeiro, as onças suçuaranas (Puma concolor) apresentam interesse por essa técnica. Neste caso foram utilizadas caixas de papelão com carne bovina para o enriquecimento alimentício, canela em pó e erva doce para o enriquecimento sensorial. Introduziram também uma bola de cipó, para avaliar a proteção e interesse. No resultado deste estudo, os machos mostraram-se receptivos pelo enriquecimento alimentício, enquanto as fêmeas pelo enriquecimento sensorial e com mais afeito a brincadeiras com outros animais. Sendo também evidenciado que os animais possuíam preferência por locais com sombra, vegetação e longe das grades.

Segundo Santos et al. (2015), foi realizado também um estudo utilizando um Guaxinim (Procyon cancrivorous) macho, adulto, que vivia no Zoológico Municipal Parque Jacarandá em Uberaba (MG), sendo realizadas técnicas de EA com folhas secas, galhos secos, folhas de bananeira e bambu. Para o enriquecimento alimentar e sensorial utilizou-se osso defumado, sorvete de frutas e verduras, couro, cobertor, bola, caixa de papelão, entre outros objetos. O resultado deste estudo tornou visível que as técnicas de enriquecimento ambiental em zoológicos são indispensáveis, uma vez que o animal mostrou uma boa relação com os itens utilizados, e passou a demonstrar comportamentos típicos da espécie.

Peixoto et al. (2019) realizaram um experimento no Aquário de São Paulo (São Paulo - SP), com dois indivíduos de tamanduás-mirins adultos, sendo um macho e uma fêmea, onde também foi possível verificar o resultado positivo do EA. Foram introduzidos no ambiente o enriquecimento alimentar, social, cognitivo, sensorial e estrutural, fazendo uso de comedouros suspensos e de labirinto, comida congelada, espelhos, chá de erva doce com canela e vinagre, balanços e também mudanças no aspecto visual do recinto. $\mathrm{O}$ resultado foi de que o macho não teve um aumento de atividade significativa registrado, enquanto a fêmea mostrou boa interação com o esse enriquecimento, mas para os dois sexos isso aumentou a interatividade entre os indivíduos, ou seja, uma maior interação social, resultando em uma avaliação positiva.

\section{Conclusão}

Tendo em vista os aspectos analisados, conclui-se que o enriquecimento ambiental é de extrema importância para a manutenção do bem-estar animal, influenciando diretamente a preservação das espécies e conservação da biodiversidade, sendo esses os princípios norteadores de um zoológico.

O uso de estímulos cognitivos, sensitivos, físicos, sociais e alimentares para explorar os sentidos e instintos do animal resultando na redução do estresse. Uma vez que a ausência de estímulos no cativeiro e fatores externos como a movimentação de pessoas intensificam o desenvolvimento de estresse, afetando diretamente a saúde animal, ocasionando mudanças fisiológicas e comportamentais, dificultando a conservação das espécies. A eficácia dos estímulos depende da execução espécieespecífica, resultando em melhor adaptação e nos benefícios em sua totalidade, reafirmando a indicação da aplicação do EA em todos os recintos.

\section{Referências}

Almeida, A. M. R., Margarido, T. C. C., Monteiro Filho, E. L. A., \& Araújo Monteiro Filho, E. L. (2008). Influência do enriquecimento ambiental no comportamento de primatas do gênero Ateles em cativeiro. Arquivos de Ciências Veterinárias e Zoologia Da UNIPAR, 11(2), 97-102.

Artigas, N. A. S., \& Fischer, M. L. (2019). Limitações no cativeiro quanto a promoção de bem-estar em primatas na percepção do visitante do Zoológico de Curitiba. Revista Brasileira de Educação Ambiental, 14(1), 49-68.

Azevedo, C. S., \& Barçante, L. (2018). Enriquecimento ambiental em zoológicos: em busca do bemestar animal. Revista Brasileira de Zoociências, 19(2). https://doi.org/10.34019/25963325.2018.v19.24708. 
Bosso, P. L. (2011). Tipos de enriquecimento. In Fundação Parque Zoológico de São Paulo.

Chiquitelli Neto, M., Kanda, C. Z., Doria, E. C., Zamarrenho, L. G., \& Gonçalves, F. H. P. (2011). Avaliação do bem-estar de um bugio (Alouatta caraya) cativo durante enriquecimento social e ambiental: indicadores comportamentais. Revista de Etologia, 10(1), 12-20.

Costa, I. D. A. (2015). Clínica e comportamento de animais selvagens. Universidade de Évora.

Damasceno, J. (2018). Enriquecimento ambiental para felinos em cativeiro: classificação de técnicas, desafios e futuras direções. Revista Brasileira de Zoociências, 19(2). https://doi.org/10.34019/25963325.2018.v19.24748.

Damy, S. B., Camargo, R. S., Chammas, R., \& Figueiredo, L. F. P. (2010). Aspectos fundamentais da experimentação animal-aplicações em cirurgia experimental. Revista Da Associação Médica Brasileira, 56(1), 103-111. https://doi.org/10.1590/s0104-42302010000100024.

Dias, J. L. C. (2003). Zoológicos e a pesquisa científica. São Paulo: Biológico, 65(1), 127-128.

Ferreira, A. S., Belato, B. C. A., Silva, D. A., Santos, J. R. C., Correa, T. H. C., Gonçalves, E. S., Leira, M. H., \& Guedes, E. (2020). Bem-estar animal no zoológico: estudo de caso do zoológico de Varginha - Parque Zoobotânico Dr. Mario Frota. Revista Agroveterinária Do Sul de Minas, 2(1), 1-9.

Flickr (2014) Disponível em: <https://www.flickr.com/photos/felidtag/15309126784>. Acesso em: 9 ago. 2020.

Foz Portal da Cidade (2019). https://foz.portaldacidade.com/noticias/cidade/parque-das-aves-ofereceblocos-de-gelo-com-frutas-para-aves-se-refrescarem-1202>. Acesso em: 10 ago. 2020.

Fundación Mona. Disponível: 〈https://fundacionmona.org/en/juegos-para-chimpances-monamaze/>. Acesso em: 8 ago. 2020.

Garcia, L. C. F., \& Bernal, F. E. M. (2015). Enriquecimento ambiental e bem-estar de animais de zoológicos. Ciência Animal, 25(1), 46-52.

Jones, K.B (2019). Disponível em: <https://wildenrichment.com/primates/how-to-build-an-enrichmentrope-ladder/>. Acesso em: 10 ago. 2020.

Leira, M. H., Reghim, L. S., Cunha, L. T., Ortiz, L. S., Paiva, C. O., Botelho, H. A., Ciacci, L. S., Braz, M. S., \& Dias, N. P. P. (2017). Bem-estar dos animais nos zoológicos e a bioética ambiental. PUBVET, 11, 545-553. https://doi.org/10.22256/pubvet.v6n11.545-553.

Manfrim, T., Santos, C. M., \& Hiroki, K. A. N. (2017). Avaliação da influência das técnicas de enriquecimento ambiental nos parâmetros comportamentais de um casal de Jaguatiricas (leopardus pardalis, Linnaeus, 1758) mantidos em cativeiro no parque do jacarandá (zoológico municipal de Uberaba, Minas Gerais). Revista Brasileira de Zoociências, 18(1), 103-120. https://doi.org/10.34019/2596-3325.2017.v18.24665.

Massari, C. H. A. L., Martins, N. O., Jozala, A. F., Grotto, D., \& Gerenutti, M. (2018). Laboratory animal welfare. Brazilian Journal of Veterinary Research and Animal Science, 55(4), e145008e145008.

OIE (World Organization for Animal Health). Introduction to the recommendations for animal welfare. Terrestrial Animal Health Code, cap. 7.1, v. 1. 2019. Disponível em: <https://www.oie.int/en/animalwelfare/animal-welfare-at-a-glance/>. Acesso em: 07 ago. 2020.

Oliveira, P. K. M., \& Carpi, L. C. (2016). Enriquecimento ambiental para Ariranha (Pteronura brasiliensis) no zoológico de Brasília. Atas de Saúde Ambiental, 4(1), 30-46.

Peixoto, F. B. G., Ambrózio, M. T. G., Colbachini, H., Padilha, F. L. A., \& Costa, F. R. (2019). Enriquecimento ambiental aplicado a tamanduás-mirins (Tamandua tetradactyla) no Aquário de São Paulo: estudo de caso. Pesquisa e Ensino Em Ciências Exatas e Da Natureza, 3(2), 119-124. https://doi.org/10.29215/pecen.v3i2.1256.

Ricci, G. Dela, Branco, C. H., Sousa, R. T., \& Titto, C. G. (2018). Efeito de diferentes técnicas de enriquecimento ambiental em cativeiro de onças suçuaranas (Puma concolor). Ciência Animal Brasileira, 19(e-47693, 2018), 1-10. https://doi.org/10.1590/1809-6891v19e-47693.

Saint Louis Zoo. Disponível em: $<$ https://www.stlzoo.org/animals/enrichmenttraining/animalenrichment\#\&gid=1\&pid=2>. Acesso 
em: 10 ago. 2020.Sanders, A., \& Feijó, A. G. S. (2007). Uma reflexão sobre animais selvagens cativos em zoológicos na sociedade atual. Congresso Internacional Transdiciplinar Ambiente e Direito, 3, 1-10.

Santos, C. M., Santos, S. M., Pizzutto, C. S., \& Custódio, A. E. I. (2015). Enriquecimento Ambiental para Guaxinim, Procyon cancrivorus (Cuvier, 1798). Bioscience Journal, 31(1), 275-282. https://doi.org/0.14393/bj-v31n1a2015-19554-.

Silva, A. (2019). Importância dos zoológicos visando o bem-estar de animais silvestres e exóticos.

Silva, A. T., \& Macêdo, M. E. A. (2013). A importância do enriquecimento ambiental para o bem estar dos animais em zoológicos. In Acervo da Iniciação Científica (Issue 2).

Silva, A. T. \& Macêdo, M. E. (2014). A importância do enriquecimento ambiental para o bem-estar dos animais em zoológicos. Centro Universitário Metodista Izabela Hendrix. 2013. Disponível em:<https://www.metodista.br/revistas/revistas-izabela/index.php/aic/article/view/501/426>. Acesso em: 8 ago. 2020.

Silva, T. B. B., Abreu, J. B., Godoy, A. C., \& Carpi, L. C. F. G. (2015). Enriquecimento ambiental para felinos em cativeiro. Atas de Saúde Ambiental, 2(3), 44-52.

Singer, P. (2013). In defense of animals: The second wave. John Wiley \& Sons.

Telles, L. F., Malm, C., Melo, M. M., Vilela, D. A. R., Lago, L. A., Silva, M. X., \& Martins, N. R. da S. (2015). Arrancamento de penas psicogênico em maritacas: haloperidol e enriquecimento ambiental. Ciência Rural, 45(6), 1099-1106. https://doi.org/10.1590/0103-8478cr20140318.

The Shape of Enrichment. Environmental Enrichment Plans, 2020. Disponível em: $<$ https://theshapeofenrichmentinc.wildapricot.org/About-Enrichment>. Acesso em: 9 ago. 2020.

\section{Histórico do artigo:}

Recebido: 2 de dezembro de 2020

Aprovado: 15 de janeiro de 2021.

Disponível online: 20 de abril de 2021.

Licenciamento: Este artigoé publicado na modalidade Acesso Aberto sob a licença Creative Commons Atribuição 4.0 (CC-BY 4.0), a qual permite uso irrestrito, distribuição, reprodução em qualquer meio, desde que $o$ autor e a fonte sejam devidamente creditados. 